\title{
Lesion Heterogeneity on High-Field Susceptibility MRI Is Associated with Multiple Sclerosis Severity
}

\author{
(DD.M. Harrison, (D) X. Li, (D) H. Liu, (DC.K. Jones, (D) B. Caffo, (DP.A. Calabresi, and (DP. van Zijl
}

\begin{abstract}
BACKGROUND AND PURPOSE: Susceptibility MR imaging contrast variations reflect alterations in brain iron and myelin content, making this imaging tool relevant to studies of multiple sclerosis lesion heterogeneity. In this study, we aimed to characterize the relationship of high-field, susceptibility contrasts in multiple sclerosis lesions to clinical outcomes.
\end{abstract}

MATERIALS AND METHODS: Twenty-four subjects with multiple sclerosis underwent 7T MR imaging of the brain, disability examinations, and a fatigue inventory. The inverse of $\mathrm{T}^{*}$ relaxation time (R2*), frequency, and relative susceptibility (from quantitative susceptibility mapping) were analyzed in 306 white matter lesions.

RESULTS: Most lesions were hypointense on R2* ( $88 \%$ without a rim, $5 \%$ with). Lesions that were hyperintense on quantitative susceptibility mapping were more frequent in relapsing-remitting than in progressive multiple sclerosis ( $54 \%$ versus $35 \%, P=.018)$. Hyperintense lesion rims on quantitative susceptibility maps were more common in progressive multiple sclerosis and patients with higher levels of disability and fatigue. Mean lesion R2* was inversely related to disability and fatigue and significantly reduced in progressive multiple sclerosis. Relative susceptibility was lower in lesions in progressive multiple sclerosis (median, -0.018 ppm; range, -0.070 to 0.022 ) than in relapsing-remitting MS (median, $-0.010 \mathrm{ppm}$; range, -0.062 to $0.052 ; P=.003$ ).

CONCLUSIONS: A progressive clinical phenotype and greater disability and fatigue were associated with lower R2* and relative susceptibility values (suggestive of low iron due to oligodendrocyte loss) and rimmed lesions (suggestive of chronic inflammation) in this multiple sclerosis cohort. Lesion heterogeneity on susceptibility MR imaging may help explain disability in multiple sclerosis and provide a window into the processes of demyelination, oligodendrocyte loss, and chronic lesion inflammation.

ABBREVIATIONS: EDSS = Expanded Disability Status Scale; MFIS = Modified Fatigue Impact Scale; MSFC = Multiple Sclerosis Functional Composite; PPMS = primary-progressive multiple sclerosis; $\mathrm{QSM}=$ quantitative susceptibility mapping; $\mathrm{R}^{*}$ = inverse of $\mathrm{T}^{\star}$ relaxation time; $\mathrm{RRMS}=$ relapsing-remitting multiple sclerosis; SPMS = secondary-progressive multiple sclerosis

Q uantification of white matter lesions on MR imaging is an effective tool for clinical care and clinical trials in multiple sclerosis. However, correlations between white matter lesions and disability remain modest, and most patients with progressive

Received October 22, 2015; accepted after revision January 4, 2016

From the Department of Neurology (D.M.H.), University of Maryland School of Medicine, Baltimore, Maryland; Departments of Neurology (D.M.H., P.A.C.), Radiology and Radiological Science (X.L., C.K.J., P.v.Z.), and Biostatistics (B.C.), Johns Hopkins University School of Medicine, Baltimore, Maryland; F.M. Kirby Research Center for Functional Brain Imaging (X.L., H.L., C.K.J., P.v.Z.), Kennedy Krieger Institute, Baltimore, Maryland; and Department of Radiology (H.L.), Guangdong Academy of Medical Sciences, Guangdong General Hospital, Guangzhou, China.

Dr Harrison conceptualized and designed the study, participated in recruitment, participated in image analysis, and was primarily responsible for data analysis and manuscript preparation. Dr Li developed the methods for image analysis and performed postacquisition image processing, in addition to editing the manuscript. Dr Liu participated in image analysis and manuscript editing. Dr Jones assisted with development of methods for image acquisition and analysis and participated in manuscript editing. Dr Caffo was involved in biostatical analyses and participated in manuscript editing. Dr Calabresi participated in study design and recruitment forms of MS continue to accumulate disability despite little change in T2 lesion burden. ${ }^{1}$ Dissociation between what is seen on MR imaging and in the clinic may, in part, be due to the lack of pathologic specificity of lesions on conventional imaging. MS lesions are quite heterogeneous, with at least 4 pathologic subtypes

and manuscript editing. Dr van Zijl participated in study design, development of image-acquisition methods, development of image-analysis methods, and manuscript editing.

This study was supported, in part, by National Institutes of Health Supplemental Grants 5P41 RR15241-09S1 and 5P41EB015909 (P. van Zijl) and Bayer Schering Pharma (P.A. Calabresi). Time for data analysis was supported by the National Institutes of Health Mentored Grant K23NS072366 (D.M. Harrison).

Please address correspondence to Daniel M. Harrison, MD, Department of Neurology, University of Maryland School of Medicine, 110 South Paca St, Third Floor, Baltimore, MD 21201; e-mail: dharrison@som.umaryland.edu

- Indicates open access to non-subscribers at www.ajnr.org

$\equiv$ Indicates article with supplemental on-line table.

Indicates article with supplemental on-line photos.

http://dx.doi.org/10.3174/ajnr.A4726 
described $^{2}$ and different stages of lesion evolution having been observed (ie, acute inflammation, chronically inactive, and chronic-active inflammation). ${ }^{3}$

Integrating susceptibility-weighted imaging into lesion analysis in MS may provide an in vivo means to evaluate lesion heterogeneity and improve specificity. Postacquisition processing of susceptibility MR imaging provides quantitative maps of the inverse of $\mathrm{T}^{*}$ relaxation time $\left(\mathrm{R} 2^{\star}\right)$ and phase images that reveal underlying tissue heterogeneity in exquisite detail, ${ }^{4}$ especially at higher magnetic fields. ${ }^{5}$ The magnetic susceptibility of brain tissue, measured by $\mathrm{R} 2^{*}$ relaxation and phase contrast, is primarily driven by tissue concentrations of myelin and iron, ${ }^{6}$ making susceptibility-weighted MR imaging quite relevant in MS. Initial studies by using this technique on high-field MR imaging in MS have attempted to quantify lesion iron content with $\mathrm{R} 2^{\star}$ and have revealed lesion patterns on phase imaging that may be indicative of acute inflammation, chronic demyelination, and iron loss or deposition. ${ }^{7-10}$ However, the nature of $\mathrm{R} 2{ }^{\star}$ and phase make interpretation of the meaning of contrast alterations difficult. Because both iron and myelin have a similar effect on the $\mathrm{R} 2{ }^{\star}$ value, differentiation of their respective impacts is not possible. ${ }^{1,12} \mathrm{Be}-$ cause phase is a nonlocal measure, phase contrast can be influenced by the magnetic susceptibility of adjacent tissues, the orientation of the head in the magnetic field, and the shape of a lesion, resulting in unreliable quantification of phase shifts and artifacts such as false rims around lesions. ${ }^{13}$

Quantitative susceptibility mapping (QSM) is a recently developed analysis technique capable of providing accurate measures of inherent tissue magnetic susceptibility without the influence of adjacent tissues, head orientation, or lesion geometry. ${ }^{13-16}$ QSM may also help differentiate the effects of myelin and iron when combined with $\mathrm{R} 2{ }^{\star} .{ }^{11,12}$ We have recently reported on MS lesion contrast patterns seen on $\mathrm{R} 2^{*}$, phase, and QSM by 7T MR imaging. ${ }^{17}$ In this article, we take this investigation further, to determine whether quantitative and qualitative lesion heterogeneity on multiparametric, high-field susceptibility MR imaging is related to clinical heterogeneity in patients with MS.

\section{MATERIALS AND METHODS \\ Approvals, Consents, and Participants}

Protocols were approved by the institutional review boards at Johns Hopkins University School of Medicine and the Kennedy Krieger Institute. Volunteers with diagnoses of relapsing-remitting (RRMS), secondary-progressive (SPMS), and primary-progressive (PPMS) MS, as assigned by their treating physicians, were prospectively recruited from the Johns Hopkins Multiple Sclerosis Center. Written, informed consent was obtained from all participants.

\section{MR Imaging Protocol and Image Analysis}

Our protocol for MR imaging acquisition and lesion analysis was previously reported in detail ${ }^{17}$ and is thus only briefly discussed here. Whole-brain MR imaging was performed on a 7T Achieva scanner (Philips Healthcare, Best, the Netherlands) with the following sequences: 3D gradient-echo and magnetizationprepared FLAIR. All images were acquired with $1.0-\mathrm{mm}$ isotropic resolution.

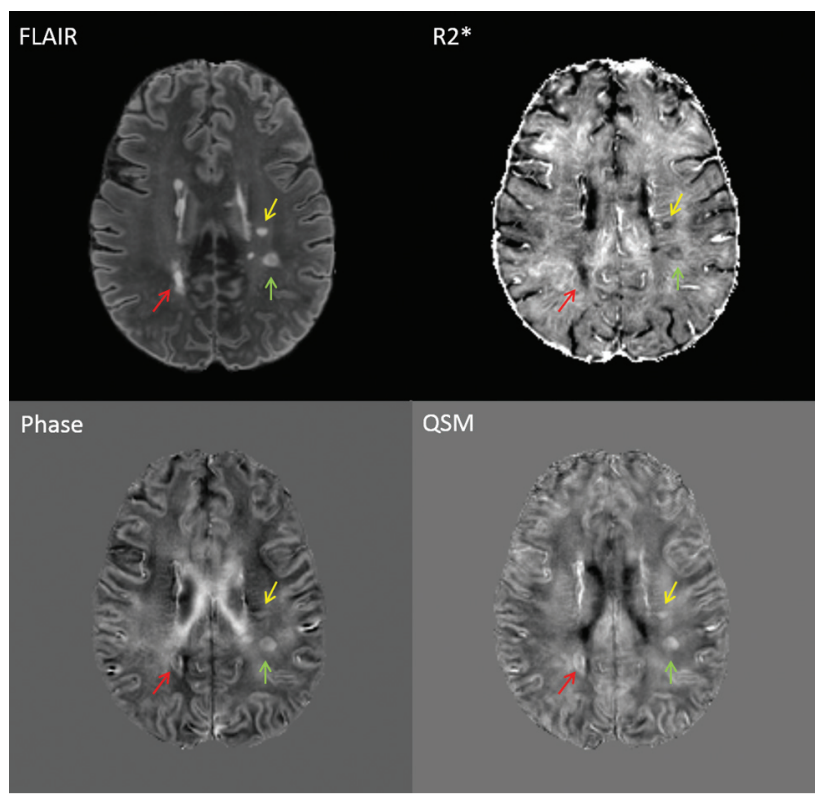

FIG 1. Examples of lesions seen on FLAIR, R2*, phase, and QSM. Each colored arrow indicates the same lesion seen on each of the 4 image contrasts. Lesions were initially identified on FLAIR images. Quantitative gray-scale values (ranging from black to white) for the above images are as follows: R2* $=0-283.78 \mathrm{~Hz}$, phase $=-48.69$ to 40.00 $\mathrm{Hz}, \mathrm{QSM}=-0.58$ to $0.40 \mathrm{ppm}$.

$3 \mathrm{D}$ gradient-echo data were processed to generate $\mathrm{R} 2^{\star}$, frequency, and susceptibility maps. Our methods for performing this task have been discussed in detail in previous publications. ${ }^{17,18}$ The effective relaxation rate $\left(\mathrm{R} 2^{*}\right)$ was estimated by fitting a monoexponential curve to the squared magnitude signal decay for all TEs by using the power method. ${ }^{1,19}$ Laplacian-based phase unwrapping ${ }^{20}$ and the V-SHARP method were used to generate the local frequency shift map. ${ }^{21}$ Dipole inversion was calculated to obtain QSM images by using the LSQR method. ${ }^{20}$ Relative susceptibility (from QSM) was calculated relative to the mean susceptibility of the central CSF region in the lateral ventricles.

Magnetization-prepared FLAIR images were coregistered to the TE 12-ms 3D-gradient recalled-echo magnitude image. A random sample of hyperintense (on magnetization-prepared FLAIR) MS lesions was chosen from each scan. Only supratentorial lesions with distinct borders and ovoid-like shapes were chosen to allow more uniform analysis. Each lesion was manually segmented on magnetization-prepared FLAIR. For each lesion, $\mathrm{R} 2^{*}$, phase, and QSM images (Fig 1) were reviewed and lesions were labeled as hypointense, isointense, or hyperintense relative to local white matter. If a lesion was found to have a rim, the intensity of the rim was described as hypointense or hyperintense relative to the lesion core and adjacent white matter. Mean values for R2*, frequency (from phase), and relative susceptibility (from QSM) in each lesion mask were calculated as a mean of all voxels within that mask, as previously described. ${ }^{17}$

\section{Disability Measures}

Expanded Disability Status Scale (EDSS) examinations were performed, and subjects were labeled as highly disabled versus less disabled by separating EDSS scores into tertiles and assigning the 
highly disabled category to the upper tertile (EDSS $\geq 5.0$ ). The Multiple Sclerosis Functional Composite (MSFC) was administered, and the National Multiple Sclerosis Society Clinical Outcomes Assessment Task Force dataset was used for normalization. $^{22,23}$ The Modified Fatigue Impact Scale (MFIS) was administered, and clinically significant fatigue was defined as MFIS $\geq 40$. $^{24,25}$

\section{Statistical Analysis}

Statistical analysis was performed in STATA 10.1 IC (StataCorp, College Station, Texas). Group differences were evaluated by $\chi^{2}$

Table 1: Demographic and clinical characteristics of study sample

\begin{tabular}{lc}
\hline \multicolumn{1}{c}{ Demographics/Characteristics } & \\
\hline No. of subjects & 24 \\
No. of lesions & 306 \\
Lesions per subject analyzed (median) & $12(2-29)$ \\
$\quad$ (range) & \\
Age (mean) (SD) (yr) & $44.3(10.0)$ \\
Sex (No.) (\%) & $12(50 \%)$ \\
$\quad$ Female & $12(50 \%)$ \\
$\quad$ Male & $11.2(7.6)$ \\
Disease duration (mean) (SD) (yr) & \\
Clinical phenotype (No.) (\%) & $21(88 \%)$ \\
$\quad$ Relapsing-remitting & $2(8 \%)$ \\
$\quad$ Secondary-progressive & $1(4 \%)$ \\
$\quad$ Primary-progressive & $19(79 \%)$ \\
On MS treatment (No.) (\%) & $3.0(1.5-6.5)$ \\
EDSS score (median) (range) & $37.7(19.3)$ \\
MFIS score (mean) (SD) & $23.0(6.6)$ seconds \\
9-HPT, dominant hand (mean) (SD) & $27.7(17.9)$ seconds \\
9-HPT, nondominant hand (mean) (SD) & $5.3(2.4)$ seconds \\
Timed 25-ft walk (mean) (SD) & $46.3(9.8)$ \\
PASAT-3 score (mean) (SD) & $-0.27(1.54)$ \\
MSFC z score (mean) (SD) &
\end{tabular}

Note:-9-HPT indicates Nine Hole Peg Test; PASAT-3, Paced Auditory Serial Addition Test, 3-second delay.

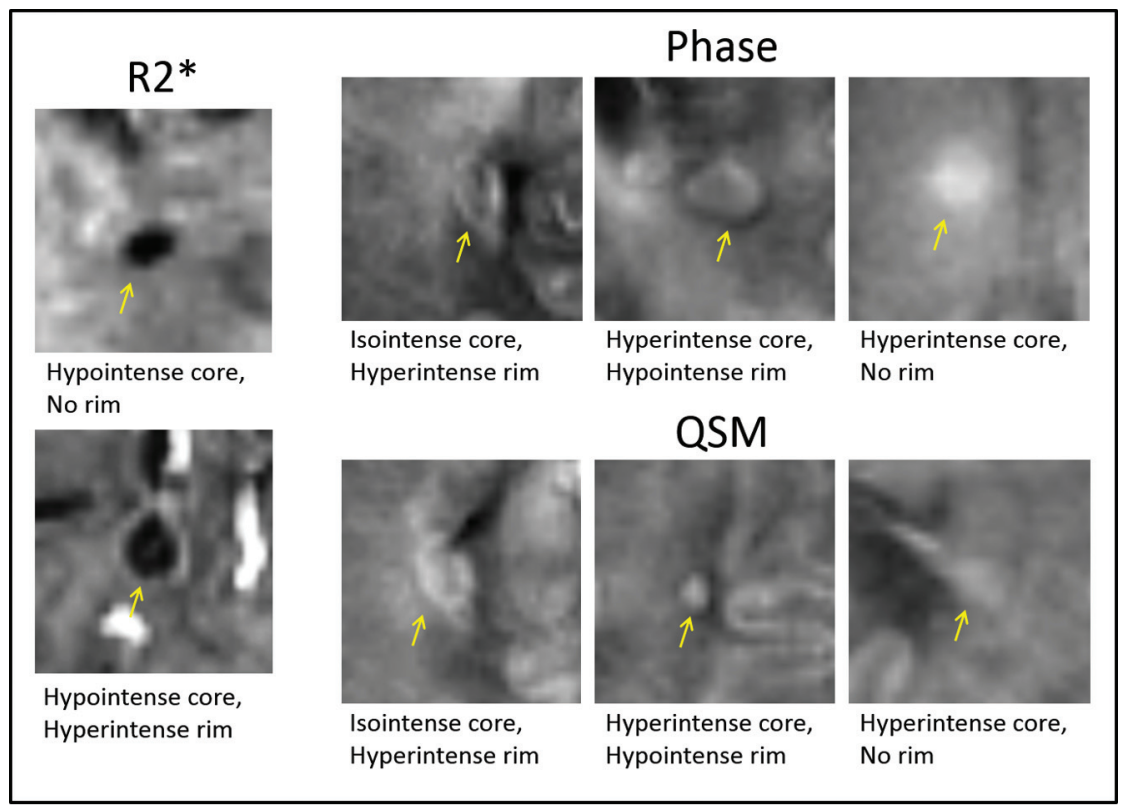

FIG 2. Examples of common lesion patterns on R2*, phase, and QSM. Yellow arrows indicate lesions identified and shown here as samples of common lesion patterns found on each image contrast. Lesions were identified by the intensity of their core and outer rim when visually compared with surrounding white matter. Only a small portion of lesions were invisible on R2* (6\%), whereas a larger proportion were invisible on phase (42\%) and QSM (36\%). (proportions) or Wilcoxon rank sum (quantitative values) tests. The relationship between quantitative MR imaging values and clinical outcomes was tested by linear mixed-effects regression. ${ }^{26}$ In addition to accounting for the fixed effects of covariates (age and sex), the random-effects portion of the regression model accounts for intrasubject correlations and intersubject variability in the number of lesions contributing to the model. Because R2*, frequency, and relative susceptibility are reported on different quantitative scales, each value was converted to a $z$ score by using the overall mean and SD. This allowed direct comparisons on a universal scale and for combining parameters through summed $z$ scores. Given the exploratory nature of this study, actual (as opposed to adjusted) $P$ values are reported throughout, allowing readers to directly assess the statistical validity of the results.

\section{RESULTS}

We reviewed scans from 24 participants with MS (21 RRMS, 2 SPMS, 1 PPMS; Table 1). Most participants were on disease-modifying therapy (79\%) at the time of their scan, and the population was moderately disabled (median EDSS score, 3.0; range, 1.5-6.5).

A total of 306 lesions were selected. Figure 2 provides examples of the commonly identified lesion patterns on $\mathrm{R} 2{ }^{*}$, phase, and QSM. The count and proportion of lesion patterns are listed in the On-line Table. Most lesions were hypointense with no rim (88\%) or had a hyperintense rim (5\%) on R2* maps. The most common patterns on phase were the following: isointense with no rim (thus invisible, 129 lesions [42\%]) and hyperintense with no rim (90 lesions [29\%]). Forty-eight (16\%) lesions were noted to have a rim on phase. The most common patterns on QSM were hyperintense with no rim (158 lesions [52\%]) and isointense with no rim (thus invisible, 110 [36\%]). Twenty-five (9\%) lesions were noted to have a rim on QSM.

No significant differences in the $\mathrm{R} 2{ }^{*}$ appearance of lesions were noted for RRMS versus progressive phenotypes (SPMS/PPMS). However, lesions with a hyperintense rim on phase were overrepresented in subjects with SPMS/ PPMS (26\% versus 11\%, $P=.006$ ). Similarly, lesions with any rim on QSM occurred more frequently in subjects with SPMS/PPMS compared with RRMS (23\% versus 6\%, $P<.001$ ). This difference was most influenced by a higher proportion of lesions with an isointense core and a hyperintense rim in those with SPMS/PPMS compared with RRMS ( $16 \%$ versus $4 \%, P=.001$ ). Conversely, hyperintense QSM lesions without any rim occurred more frequently in subjects with RRMS compared with SPMS/PPMS (54\% versus $35 \%, P=.018)$.

In subjects with more severe disability (EDSS $\geq 5.0$ ), lesions were more likely to be hypointense with no rim on 
RRMS SPMS/PPMS
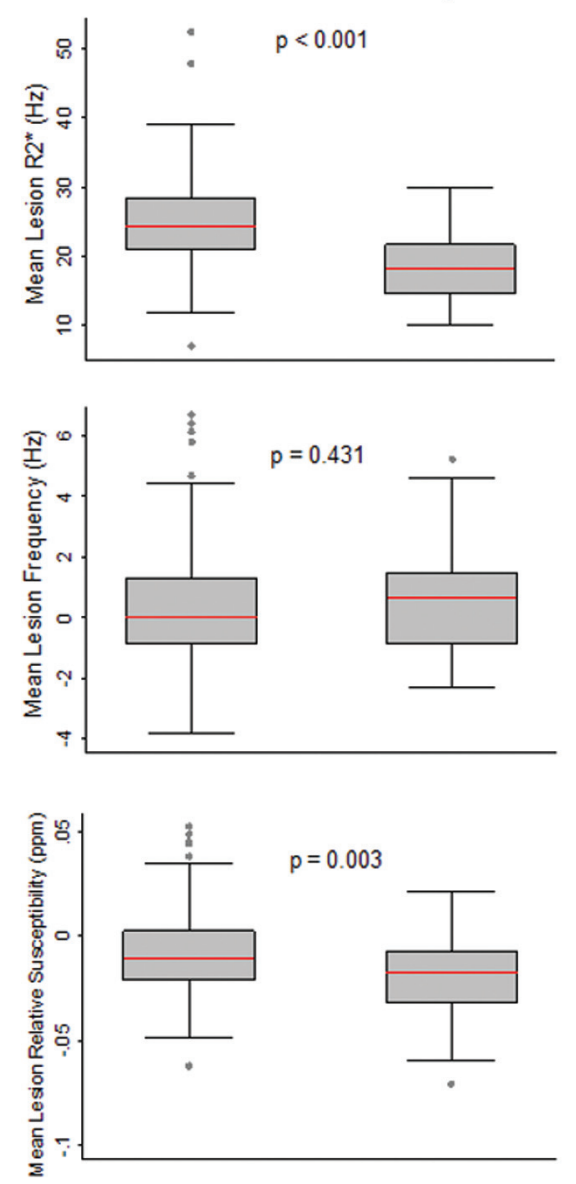

EDSS $<5.0 \quad$ EDSS $\geq 5.0$
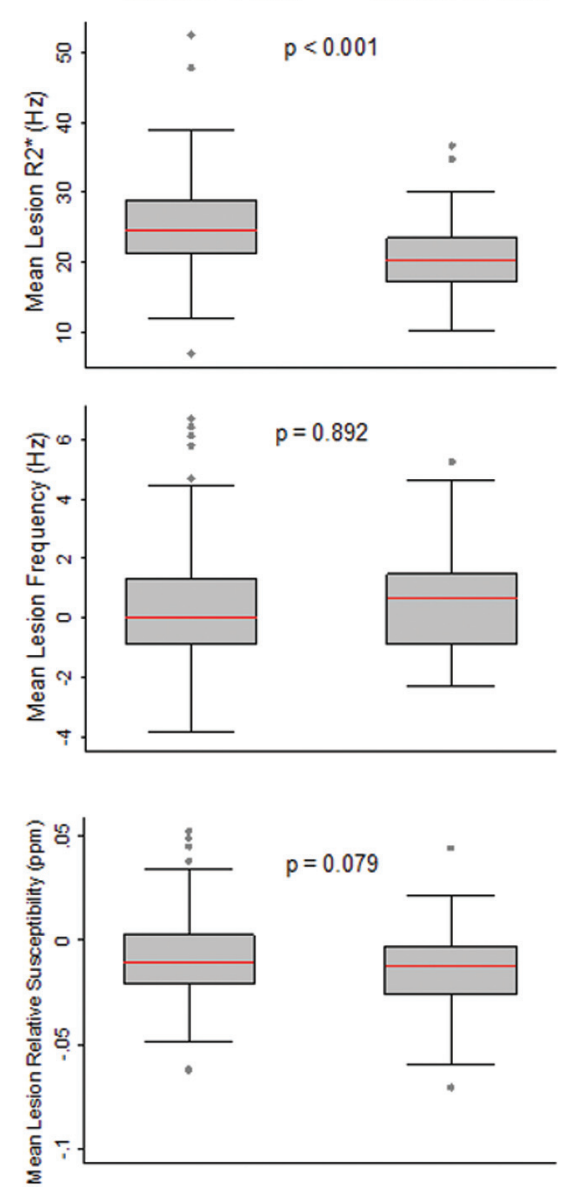

FIG 3. Quantitative comparison of lesion susceptibility values by disease subtype and level of disability. Box-and-whisker plots showing a quantitative comparison of mean lesion R2*, frequency (from phase), and relative susceptibility (from QSM) between subjects with RRMS and SPMS/PPMS (left column) and those with lower-versus-higher levels of disability based on the EDSS score (right column). Lesion R2* values were significantly lower in SPMS/PPMS and EDSS $\geq$ 5.0 , and mean lesion relative susceptibility was lower in SPMS/PPMS. Lesion values were taken as the mean of all voxels within each lesion. Red lines indicate the median value for all lesions in each group. $P$ values represent the results of Wilcoxon rank sum testing.

$\mathrm{R} 2 *$ (95\% versus $85 \%, P=.015)$. Lesions with an isointense core and hyperintense rim on phase and QSM were also noted more commonly in more severely disabled subjects and in those with clinically significant fatigue compared with those with lower disability (EDSS $<5.0$ ) or without fatigue.

Mean lesion R2* was significantly lower in patients with SPMS/PPMS or EDSS $\geq 5.0$ (Fig 3). Mean relative susceptibility (from QSM) in lesions was significantly lower in subjects with SPMS/PPMS (median, $-0.018 \mathrm{ppm}$; range, -0.070 to 0.022 ) compared with RRMS (median, $-0.010 \mathrm{ppm}$; range, -0.062 to 0.052; $P=.003)$.

In a multivariate linear mixed-model regression, mean lesion $\mathrm{R} 2{ }^{\star}$ had a significant inverse relationship with the EDSS score (Fig 4). No significant relationships were found between EDSS and mean lesion frequency or relative susceptibility. However, mean lesion $\mathrm{R} 2{ }^{*}$ and relative susceptibility values combined (summed $z$ scores) had a significant inverse relationship with EDSS (Fig 4). The magnitude of the regression coefficient for the relationship between EDSS and $\mathrm{R} 2{ }^{*}+$ relative susceptibility was greater than that for the $\mathrm{R} 2^{*}$ value alone: $-0.307(-0.536,-0.079)$ versus $-0.232(-0.366,-0.097)$, respectively.

Mean lesion R2* values were positively related to the MSFC score $(P=$ .012 , On-line Fig 1), and the combined index of $\mathrm{R} 2^{*}+$ relative susceptibility was positively related to the MSFC score $(P=.010)$.

Mean lesion R2* values were inversely related to the MFIS score $(P=$ .039, On-line Fig 2). No significant relationships were found between MFIS scores and mean lesion frequency, relative susceptibility, or the combination of either with R2*.

\section{DISCUSSION}

Our results indicate that multiparametric susceptibility $M R$ imaging reveals heterogeneity of white matter lesions in MS and this lesion heterogeneity is related to the clinical heterogeneity seen in patients. Although previous work has shown heterogeneity in MS lesions on phase contrast, we found changes on $\mathrm{R} 2^{*}$ and QSM to be most clinically relevant. ${ }^{8,9}$ Subjects with RRMS were more likely to have hyperintense lesions with no rim on QSM, whereas lesions with isointense cores and hyperintense rims on phase and QSM were more likely in subjects with SPMS/PPMS, greater disability, or fatigue. Progressive phenotypes of MS and greater disability and fatigue were also associated with lower $\mathrm{R} 2^{\star}$ and relative susceptibility values in lesions. These results suggest that multiparametric susceptibility MR imaging provides clinically relevant characterization of MS lesions.

The possible pathologic basis of the observed lesion patterns and quantification of magnetic susceptibility is informed by prior histopathologic and imaging correlative studies. Most of the magnetic susceptibility signal in the human brain is determined by tissue concentrations of myelin and iron, along with a contribution from the anisotropic properties of tissue architecture. ${ }^{11,12,18,27}$ Histopathologic myelin content is positively correlated with $\mathrm{R} 2{ }^{\star}$ and inversely correlated with relative susceptibility (due to diamagnetic properties). ${ }^{11,12}$ Thus, demyelination results in a reduced $\mathrm{R} 2{ }^{*}$ value and an elevation of relative susceptibility. Iron, on the other hand, has a paramagnetic effect on relaxation and susceptibility, causing both the $\mathrm{R} 2^{*}$ value and relative susceptibility to increase with iron deposition. ${ }^{11,12}$ The effect of changes in myelin and iron content on phase is more difficult to interpret, likely due to sensitivity to tissue architecture and nonlocal phase effects. $^{7,8,28}$

Given the parallel effects of myelin and iron on $\mathrm{R} 2^{\star}$ and the 

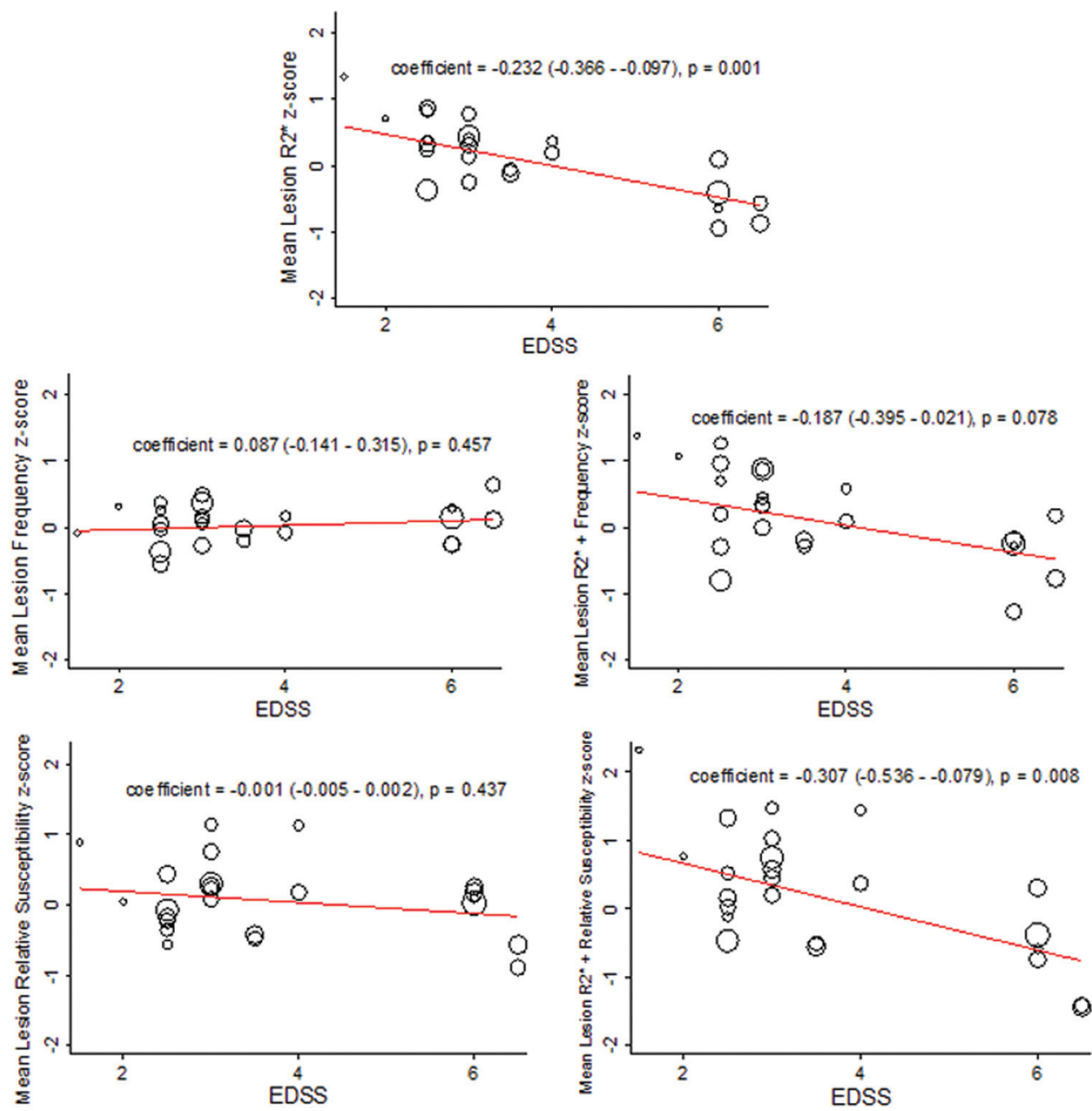

FIG 4. Relationship between EDSS and quantitative R2*, frequency, and relative susceptibility values. Shown are the results of linear mixedmodel regression (adjusted for age and sex) for prediction of the quantitative MR imaging index value by the Expanded Disability Status Scale score as represented by a fitted-values plot. A significant inverse relationship was found between R2* and EDSS and for a combined index of $\mathrm{R} 2 *+$ relative susceptibility and EDSS. The open circles represent fitted values based on the fixed and random effects from the model, with each circle thus representing 1 subject and the size of the circle weighted for the number of lesions that particular subject contributed to the model. The regression coefficient for the fixed-effects portion of the model is shown in each panel, along with the $P$ value for the significance of that coefficient. To place all quantitative values in an equivalent space, we converted all values to $z$ score units (based on mean and SD from all lesions).

Table 2: Hypothesized alterations in myelin and iron content in MS lesions associated with commonly observed R2*/QSM lesion contrast patterns

\begin{tabular}{lccc}
\hline R2* Intensity & QSM Intensity & $\begin{array}{c}\text { Alteration in } \\
\text { Myelin Content }\end{array}$ & $\begin{array}{c}\text { Alteration in } \\
\text { Iron Content }\end{array}$ \\
\hline Hypo & Hyper & $\downarrow$ & $\leftrightarrow$ \\
Hypo & Iso & $\downarrow$ & $\downarrow$ \\
Iso & Hyper & $\downarrow$ & $\uparrow$ \\
\hline
\end{tabular}

Note:- Hypo indicates hypointensity; Hyper, hyperintensity; Iso, isointensity; $\uparrow$, increase; $\downarrow$, decrease; $\leftrightarrow$, little-to-no change.

opposing effects of myelin and iron on QSM, analyzing both in tandem may help separate the influence of myelin and iron on magnetic susceptibility (Table 2). ${ }^{11,12,17}$ The overwhelming majority of lesions in this study were hypointense on $\mathrm{R} 2{ }^{*}$, which would indicate either a loss of myelin, a reduction in iron, or both. Pathology-imaging correlation studies have confirmed that most lesions with low $\mathrm{R}^{*}$ have nearly absent myelin staining and low iron content. ${ }^{7}$ A lesion with low $\mathrm{R} 2{ }^{\star}$ being hyperintense on QSM would indicate that most of the signal change is due to myelin loss without a meaningful change in iron content. On the other hand, a lesion hypointense on $\mathrm{R}^{*}$ and isointense on QSM may indicate a lesion with reductions in both iron and myelin content. Most of the iron found in normal white matter is bound to ferritin within oligodendrocytes and oligodendrocyte progenitor cells, ${ }^{7,29}$ in which iron is necessary for enzymatic functions, including those involved with myelin lipid biosynthesis. ${ }^{30}$ A smaller amount of iron can be found within myelin sheaths and microglia. ${ }^{7}$ Loss of iron from MS lesions is thus most likely to indicate a significant reduction in oligodendrocytes, which has been confirmed pathologically. ${ }^{7}$

Knowledge of the underlying pathologic causes of $\mathrm{R} 2^{*}$ and QSM signal changes may allow stratification of the lesion patterns observed in this study along a similar classification scheme as proposed by Lucchinetti et al. ${ }^{2}$ Lesions that were hypointense on R2* and hyperintense on QSM likely correspond to type I and II white matter lesions, in which there is demyelination, some remyelination, and little change in oligodendrocytes. We found this lesion type more prominently in RRMS. Type III and IV lesions have demyelination, no remyelination, and a loss of oligodendro- 
cytes through apoptotic mechanisms, all likely making these lesion subtypes of greater clinical consequence. A higher proportion of lesions of these subtypes would be expected to manifest lower mean $\mathrm{R}^{*}$ and less increased relative susceptibility values, indicating more severe demyelination and iron loss. We found this lesion pattern associated with progressive MS and more disability and fatigue.

Lucchinetti et $\mathrm{al}^{2}$ also described a rim of active destruction of oligodendrocytes and demyelination occurring in a ring of periplaque white matter in type IV lesions, a lesion subtype only seen in PPMS in their study. It is possible that type IV lesions were being observed in their study, with lesion rims resulting in signal alterations through the release of intracellular iron stores as oligodendrocytes are destroyed and/or a rim of severe demyelination. This possibility would be consistent with our finding of a higher proportion of visible lesion rims on phase and QSM in patients with a progressive phenotype, higher levels of disability, and greater fatigue.

The presence of lesions with and without observable rims may also be indicative of the stage of evolution of a lesion. Histopathology allows temporal staging of lesions as acute inflammatory, chronic inflammatory/slowly expanding, and chronic-inactive. ${ }^{3}$ The infiltration of inflammatory cells into acutely inflamed MS lesions leads to a temporary increase in iron content due to enzymatic activity in microglia and macrophages ${ }^{7}$ and release of intracellular stores from injured oligodendrocytes and myelin sheaths. ${ }^{31}$ Acutely increased iron at the same time as active demyelination would result in a counterbalancing effect on $\mathrm{R} 2^{*}$ and an additive effect to increase magnetic susceptibility. An early increase in frequency (from phase) and relative susceptibility (from QSM) has been previously described in longitudinal lesion studies, with subsequent decrease as free iron is scavenged and remyelination occurs. ${ }^{27,32}$ This would explain our finding of a higher proportion of hyperintense lesions on QSM and higher R2* and relative susceptibility values in patients with RRMS (in whom lesions are more likely to be in an early stage of development) compared with those with progressive disease.

On the other hand, lesions with an isointense core and a hyperintense rim may represent slowly expanding, chronically inflamed lesions. Activated microglia at the rim of a lesion is a pathologic hallmark of lesions that are gradually expanding due to chronic inflammation. Slowly expanding lesions with activated microglia are seen more commonly in patients with progressive $\mathrm{MS}^{3}$ and have been proposed as a mechanism for progressive disability accumulation. ${ }^{33}$ In chronic-active lesions, CD68 staining (microglia, macrophages) colocalizes with iron and ferritin at the outer rim of lesions, consistent with iron-rich, M1-polarized, nonphagocytic macrophages. ${ }^{7,34}$ Iron uptake at the lesion rim enhances M1 polarization, resulting in proinflammatory tumor necrosis factor $\alpha$ and inducible nitric oxide synthase release and inhibition of the phagocytic M2 macrophage phenotype, which is necessary for tissue repair. ${ }^{34}$ Lesions of this type also have shown reductions in oligodendrocytes and no oligodendrocyte progenitor (NG2) cells, further inhibiting tissue repair. ${ }^{35}$ The expected myelin and iron properties of chronic-active lesions are consistent with those in lesions that are R2* hypointense and isointense with a hyperintense rim on QSM, which were found in a higher pro- portion in subjects with progressive disease and greater disability and fatigue in this study.

Although lesion rims were found in higher proportion on phase images compared with QSM, lesion rims seen on these 2 contrasts may not have the same pathologic meaning. Lesion modeling data have shown that the geometry of a lesion can lead to false classification of solid lesions as shell-shaped on phase, which is not the case for QSM. ${ }^{13}$ Furthermore, due to magnetic dipole effects, phase rims can appear as hyper- or hypointense, depending on orientation, in addition to being influenced by adjacent structures such as veins and crossing fiber tracts. ${ }^{13} \mathrm{Al}-$ though previous publications have suggested that the presence of rims on phase may represent blood-brain barrier breakdown in acute lesions, ${ }^{9}$ subsequent data have shown that this change is more likely due to a transition from anisotropic to isotropic tissue architecture, along with some false rims. ${ }^{13,27}$ Our analysis of phase images furthers the argument of the benefits of QSM over phase. In addition to the potential presence of false lesion rims, we found a higher proportion of lesions that were invisible on phase, and lesion frequency was not related to disability measures.

This study has limitations. The lack of contrast administration or longitudinal follow-up tempers our conclusions as to the relationship between $\mathrm{R} 2 *$ and QSM findings and inflammatory states and lesion evolution. Furthermore, although our conclusions as to the pathologic meaning of susceptibility signal alterations in lesions are supported by prior histopathologic-imaging studies, without postmortem data in this cohort, such conclusions can only be speculative. In addition, although the total number of lesions evaluated here were quite high, only 24 subjects were assessed, which limits conclusions on relapsing-versus-progressive MS. Further stratification of subjects with progressive disease into primary- and secondary-progressive may also have yielded further pathologic insight, but it was not possible due to the small sample size. The restriction of lesion analysis to individual ovoid lesions can also potentially be criticized. Before conclusions can be drawn on the utility of susceptibility imaging in MS, future work should investigate the meaning of susceptibility alterations in larger areas of lesion formation, normal-appearing white matter, cortical gray matter, and other structures. The results reported here should thus be considered preliminary, with further work necessary to confirm or refute these conclusions. Our planned future work will include administration of contrast agents, follow-up scanning, and recruitment of a larger cohort.

\section{CONCLUSIONS}

Despite limitations, we believe that our findings have profound implications for MS research. Our results imply that combined analysis of R2* and QSM imaging may provide an in vivo probe into tissue myelin and iron content and thus into the impact of white matter inflammation on axonal tracts and oligodendrocytes. We have also shown that R2* and QSM analysis can provide qualitative and quantitative measures of the lesion heterogeneity, which explain the varying clinical manifestations of MS. Use of such tools is necessary to gain a greater understanding of the mechanisms of disability progression in MS and may hold promise as imaging outcome measures for trials of remyelinating and neuroprotective medications. 


\section{ACKNOWLEDGMENTS}

We thank Janelle Aquino, Michaela Seigo, Stephanie Syc, and Anna Whetstone and the dedicated Kirby Center staff.

Disclosures: Daniel M. Harrison—RELATED: Grant: National Institutes of Health*; Support for Travel to Meetings for the Study or Other Purposes: National Institutes of Health*; UNRELATED: Consultancy: Mallinckrodt Pharmaceuticals, Genzyme, EMD Serono, Medlmmune; Grants/Grants Pending: Bayer, ${ }^{*}$ EMD Serono, ${ }^{*}$ National Multiple Sclerosis Society *; OTHER: Dr Harrison has received research support from Bayer Neurosciences and EMD Serono and has received consulting fees from Mallinckrodt Pharmaceuticals, Genzyme, EMD Serono, and Medlmmune. Craig K. Jones—RELATED: Grant: Philips Healthcare, ${ }^{*}$ Comments: I was previously paid, in part, from a grant from Philips Healthcare to Kennedy Krieger Institute. Brian CaffoRELATED: Grant: National Institutes of Health. * Peter A. Calabresi-RELATED: Grant: Bayer*; UNRELATED: Consultancy: Vertex, Vaccinex, Merck, Prothena; Grants/ Grants Pending: Novartis, ${ }^{*}$ Medlmmune, ${ }^{*}$ Biogen Idec*; Payment for Lectures (including service on Speakers Bureaus): Multiple Sclerosis Association of America, Vindico Communications; OTHER: Dr Calabresi has received research funding from Biogen Idec, Vertex, Novartis, Medlmmune, and Bayer and honoraria for consulting from Vaccinex, Vertex, Abbott Laboratories, Merck, and Prothena. Peter van Zijl— RELATED: Grant: Philips Healthcare*; UNRELATED: Grants/Grants Pending: Philips Healthcare*; Payment for Lectures (including service on Speakers Bureaus): Philips Healthcare*; Patents (planned, pending, or issued): Philips Healthcare, ${ }^{*}$ Comments: They licensed some of my patents; Royalties: Philips Healthcare, ${ }^{\star}$ Comments: from patent licensing; Travel/Accommodations/Meeting Expenses Unrelated to Activities Listed: Philips Healthcare.* *Money paid to the institution.

\section{REFERENCES}

1. Barkhof F. MRI in multiple sclerosis: correlation with expanded disability status scale (EDSS). Mult Scler 1999;5:283-86 CrossRef Medline

2. Lucchinetti C, Brück W, Parisi J, et al. Heterogeneity of multiple sclerosis lesions: implications for the pathogenesis of demyelination. Ann Neurol 2000;47:707-17 Medline

3. Kutzelnigg A, Lucchinetti CF, Stadelmann C, et al. Cortical demyelination and diffuse white matter injury in multiple sclerosis. Brain 2005;128(pt 11):2705-12 CrossRef Medline

4. Rauscher A, Sedlacik J, Barth M, et al. Magnetic susceptibilityweighted MR phase imaging of the human brain. AJNR Am J Neuroradiol 2005;26:736-42 Medline

5. Deistung A, Rauscher A, Sedlacik J, et al. Susceptibility weighted imaging at ultra high magnetic field strengths: theoretical considerations and experimental results. Magn Reson Med 2008;60: 1155-68 CrossRef Medline

6. Langkammer C, Krebs N, Goessler W, et al. Susceptibility induced gray-white matter MRI contrast in the human brain. Neuroimage 2012;59:1413-19 CrossRef Medline

7. Bagnato F, Hametner S, Yao B, et al. Tracking iron in multiple sclerosis: a combined imaging and histopathological study at 7 Tesla. Brain 2011;134(pt 12):3602-15 CrossRef Medline

8. Yao B, Bagnato F, Matsuura E, et al. Chronic multiple sclerosis lesions: characterization with high-field-strength MR imaging. $\mathrm{Ra}$ diology 2012;262:206-15 CrossRef Medline

9. Absinta M, Sati P, Gaitán MI, et al. Seven-Tesla phase imaging of acute multiple sclerosis lesions: a new window into the inflammatory process. Ann Neurol 2013;74:669-78 CrossRef Medline

10. Hammond KE, Metcalf $M$, Carvajal $L$, et al. Quantitative in vivo magnetic resonance imaging of multiple sclerosis at 7 Tesla with sensitivity to iron. Ann Neurol 2008;64:707-13 CrossRef Medline

11. Deistung A, Schäfer A, Schweser F, et al. Toward in vivo histology: a comparison of quantitative susceptibility mapping (QSM) with magnitude-, phase-, and R2*-imaging at ultra-high magnetic field strength. Neuroimage 2013;65:299-314 CrossRef Medline

12. Stuber C, Morawski M, Schäfer A, et al. Myelin and iron concentration in the human brain: a quantitative study of MRI contrast. Neuroimage 2014;93(pt 1):95-106 CrossRef Medline

13. Eskreis-Winkler S, Deh K, Gupta A, et al. Multiple sclerosis lesion geometry in quantitative susceptibility mapping (QSM) and phase imaging. J Magn Reson Imaging 2015;42:224-29 CrossRef Medline
14. Liu C, Li W, Tong KA, et al. Susceptibility-weighted imaging and quantitative susceptibility mapping in the brain. J Magn Reson Imaging 2015;42:23-41 CrossRef Medline

15. Wang Y, Liu T. Quantitative susceptibility mapping (QSM): decoding MRI data for a tissue magnetic biomarker. Magn Reson Med 2015;73:82-101 CrossRef Medline

16. Wharton S, Bowtell R. Whole-brain susceptibility mapping at high field: a comparison of multiple- and single-orientation methods. Neuroimage 2010;53:515-25 CrossRef Medline

17. Li X, Harrison DM, Liu H, et al. Magnetic susceptibility contrast variations in multiple sclerosis lesions. J Magn Reson Imaging 2016; 43:463-73 CrossRef Medline

18. Li X, Vikram DS, Lim IA, et al. Mapping magnetic susceptibility anisotropies of white matter in vivo in the human brain at $7 \mathrm{~T}$. Neuroimage 2012;62:314-30 CrossRef Medline

19. Miller AJ, Joseph PM. The use of power images to perform quantitative analysis on low SNR MR images. Magn Reson Imaging 1993; 11:1051-56 CrossRef Medline

20. Li W, Wu B, Liu C. Quantitative susceptibility mapping of human brain reflects spatial variation in tissue composition. Neuroimage 2011;55:1645-56 CrossRef Medline

21. Li W, Avram AV, Wu B, et al. Integrated Laplacian-based phase unwrapping and background phase removal for quantitative susceptibility mapping. NMR Biomed 2014;27:219-27 CrossRef Medline

22. Fischer JS, Rudick RA, Cutter GR, et al. The Multiple Sclerosis Functional Composite Measure (MSFC): an integrated approach to MS clinical outcome assessment-National MS Society Clinical Outcomes Assessment Task Force. Mult Scler 1999;5:244-50 CrossRef Medline

23. Rudick R, Antel J, Confavreux C, et al. Recommendations from the National Multiple Sclerosis Society Clinical Outcomes Assessment Task Force. Ann Neurol 1997;42:379-82 CrossRef Medline

24. Flachenecker P, Kümpfel T, Kallmann B, et al. Fatigue in multiple sclerosis: a comparison of different rating scales and correlation to clinical parameters. Mult Scler 2002;8:523-26 CrossRef Medline

25. Achiron A, Givon U, Magalashvili D, et al. Effect of Alfacalcidol on multiple sclerosis-related fatigue: a randomized, double-blind placebo-controlled study. Mult Scler 2015;21:767-75 CrossRef Medline

26. Laird NM, Ware JH. Random-effects models for longitudinal data. Biometrics 1982;38:963-74 CrossRef Medline

27. Wiggermann V, Hernandez Torres E, Vavasour IM, et al. Magnetic resonance frequency shifts during acute MS lesion formation. $\mathrm{Neu}$ rology 2013;81:211-18 CrossRef Medline

28. Yablonskiy DA, Luo J, Sukstanskii AL, et al. Biophysical mechanisms of MRI signal frequency contrast in multiple sclerosis. Proc Natl Acad Sci U S A 2012;109:14212-17 CrossRef Medline

29. Hulet SW, Heyliger SO, Powers S, et al. Oligodendrocyte progenitor cells internalize ferritin via clathrin-dependent receptor mediated endocytosis. J Neurosci Res 2000;61:52-60 Medline

30. Connor JR, Menzies SL. Relationship of iron to oligodendrocytes and myelination. Glia 1996;17:83-93 Medline

31. Hametner S, Wimmer I, Haider L, et al. Iron and neurodegeneration in the multiple sclerosis brain. Ann Neurol 2013;74:848-61 CrossRef Medline

32. Chen W, Gauthier SA, Gupta A, et al. Quantitative susceptibility mapping of multiple sclerosis lesions at various ages. Radiology 2014;271:183-92 CrossRef Medline

33. Prineas JW, Kwon EE, Cho ES, et al. Immunopathology of secondary-progressive multiple sclerosis. Ann Neurol 2001;50:646-57 CrossRef Medline

34. Mehta V, Pei W, Yang G, et al. Iron is a sensitive biomarker for inflammation in multiple sclerosis lesions. PLoS One 2013;8:e57573 CrossRef Medline

35. Chang A, Nishiyama A, Peterson J, et al. NG2-positive oligodendrocyte progenitor cells in adult human brain and multiple sclerosis lesions. J Neurosci 2000;20:6404-12 Medline 